\title{
Optimal Quantum Tomography of States, Measurements, and Transformations
}

\author{
A. Bisio, G. Chiribella, G. M. D'Ariano, S. Facchini, and P. Perinotti \\ Quit group, Dipartimento di Fisica "A. Volta", via Bassi 6, I-27100 Pavia, Italy and CNISM.
}

\begin{abstract}
We present the first complete optimization of quantum tomography, for states, POVMs, and various classes of transformations, for arbitrary prior ensemble and arbitrary representation, giving corresponding feasible experimental schemes.
\end{abstract}

A crucial issue in quantum information theory is the precise determination of states and processes. The procedure by which this task can be accomplished is known as quantum tomography [1, 2, 3].

The most general quantum measurement is described by a POVM, namely a collection of positive operators $P_{i} \in \mathcal{B}(\mathcal{H})$ satisfying the normalization $\sum_{i} P_{i}=I[4]$. The probability distribution of the outcome $i$ of the measurement is provided by the Born statistical formula

$$
p_{i}=\operatorname{Tr}\left[\rho P_{i}\right] .
$$

Tomographing an unknown state $\rho$ of a quantum system means performing a suitable POVM $\left\{P_{i}\right\}$ such that every expectation value can be evaluated from the probability distribution $p_{i}=\operatorname{Tr}\left[\rho P_{i}\right]$. In particular the expectation value of an operator $A$ can be obtained when it is possible to expand $A$ over the POVM as follows

$$
A=\sum_{i} f_{i}[A] P_{i}
$$

$f_{i}[A]$ denoting suitable expansion coefficients. The expectation of $A$ is then obtained as $\langle A\rangle=\sum_{i} f_{i}[A]\left\langle P_{i}\right\rangle$. When expansion (21) holds for all operators $\mathcal{B}(\mathcal{H})$-i. e. $\mathcal{B}(\mathcal{H})=\operatorname{span}\left\{P_{i}\right\}$ - the POVM is called informationally complete $[5,6]$.

It is convenient to associate every operator $A \in \mathcal{B}(\mathcal{H})$ to a bipartite vector in $\mathcal{H} \otimes \mathcal{H}$ in the following way

$$
\left.A=\sum_{m, n=1}^{d} A_{m n}|m\rangle\langle n|\leftrightarrow| A\rangle\right\rangle=\sum_{m, n=1}^{d} A_{m n}|m\rangle|n\rangle .
$$

Information-completeness of the POVM along with convergence of the series (2) rewrite as follows

$$
a\|A\|_{2}^{2} \leq \sum_{i=1}^{N}\left|\left\langle\left\langle P_{i} \mid A\right\rangle\right\rangle\right|^{2} \leq b\|A\|_{2}^{2}, \quad A \in B(\mathcal{H}),
$$

with $0<a \leq b<\infty$. Sets of vectors $\left.\left|P_{i}\right\rangle\right\rangle$ satisfying condition (4) are known as frames [7]. This condition is equivalent to invertibility of the frame operator $F=$ $\left.\sum_{i}\left|P_{i}\right\rangle\right\rangle\left\langle P_{i}\right|$. The expansion in Eq. (2) can be written as follows

$$
\left.|A\rangle\rangle=\sum_{i}\left\langle\left\langle D_{i} \mid A\right\rangle\right\rangle\left|P_{i}\right\rangle\right\rangle
$$

in terms of a dual frame $\left\{D_{i}\right\}$, namely a set of operators satisfying the identity $\left.\sum_{i}\left|P_{i}\right\rangle\right\rangle\left\langle\left\langle D_{i}\right|=I\right.$. For linearly dependent frame $\left\{P_{i}\right\}$ the dual $\left\{D_{i}\right\}$ is not unique.

The request for the POVM $\left\{P_{i}\right\}$ to be informationally complete can be relaxed if we have some prior information about the state $\rho$. If we know that the state belongs to a given subspace $\mathcal{V} \subseteq \mathcal{B}(\mathcal{H})$ the expectation value is

$$
\langle A\rangle=\langle\langle\rho \mid A\rangle\rangle=\left\langle\left\langle\rho\left|Q_{\mathcal{V}}\right| A\right\rangle\right\rangle
$$

$Q_{\mathcal{V}}$ orthogonal projector on $\mathcal{V}$, whence the set $\left\{P_{i}\right\}$ is required to span only $\mathcal{V}$.

For the estimation of the expectation $\langle A\rangle$ of an observable $A$, optimality means minimization of the cost function given by the variance $\delta(A)$ of the random variable $\left\langle\left\langle D_{i} \mid A\right\rangle\right\rangle$ with probability distribution $\operatorname{Tr}\left[\rho P_{i}\right]$, namely

$$
\delta(A):=\left.\sum_{i}\left|\left\langle D_{i} \mid A\right\rangle\right\rangle\right|^{2} \operatorname{Tr}\left[\rho P_{i}\right]-|\operatorname{Tr}[\rho A]|^{2} .
$$

In a Bayesian scheme the state $\rho$ is randomly drawn from an ensemble $\mathcal{S}=\left\{\rho_{k}, p_{k}\right\}$ of states $\rho_{k}$ with prior probability $p_{k}$, with the variance averaged over $\mathcal{S}$, leading to

$$
\delta_{\mathcal{S}}(A):=\sum_{i}\left|\left\langle\left\langle D_{i} \mid A\right\rangle\right\rangle\right|^{2} \operatorname{Tr}\left[\rho_{\mathcal{S}} P_{i}\right]-\sum_{k} p_{k}\left|\operatorname{Tr}\left[\rho_{k} A\right]\right|^{2}
$$

where $\rho_{\mathcal{S}}=\sum_{k} p_{k} \rho_{k}$. Moreover, a priori we can be interested in some observables more than other ones, and this can be specified in terms of a weighted set of observables $\mathcal{G}=\left\{A_{n}, q_{n}\right\}$, with weight $q_{n}>0$ for the observable $A_{n}$. Averaging over $\mathcal{G}$ we have

$$
\delta_{\mathcal{S}, \mathcal{G}}:=\sum_{i}\left\langle\left\langle D_{i}|G| D_{i}\right\rangle\right\rangle \operatorname{Tr}\left[\rho_{\mathcal{S}} P_{i}\right]-\sum_{k, n} p_{k} q_{n}\left|\operatorname{Tr}\left[\rho_{k} A_{n}\right]\right|^{2}
$$

where $\left.G=\sum_{n} q_{n}\left|A_{n}\right\rangle\right\rangle\left\langle\left\langle A_{n}\right|\right.$. The weighted set $\mathcal{G}$ yields a representation of the state, given in terms of the expectation values. The representation is faithful when $\left\{A_{n}\right\}$ is an operator frame, e. g. when it is made of the dyads $|i\rangle\langle j|$ corresponding to the matrix elements $\langle j|\rho| i\rangle$.

Notice that only the first term of $\delta_{\mathcal{S}, \mathcal{G}}$ depends on $\left\{P_{i}\right\}$ and $\left\{D_{i}\right\}$. If $\rho_{i} \in \mathcal{V}$ for all states $\rho_{i} \in \mathcal{S}$, reminding Eq. (6) the first term of Eq. (9) becomes

$$
\eta=\sum_{i}\left\langle\left\langle D_{i}\left|Q_{\mathcal{V}} G Q_{\mathcal{V}}\right| D_{i}\right\rangle\right\rangle \operatorname{Tr}\left[\rho_{\mathcal{S}} P_{i}\right]
$$

We now generalize this approach to tomography of quantum operations, keeping generally different input 
and output Hilbert spaces $\mathcal{H}_{\text {in }}$ and $\mathcal{H}_{\text {out }}$, respectively. This has the advantage that the usual tomography of states comes as the special case of one-dimensional $\mathcal{H}_{\text {in }}$, whereas tomography of POVMs corresponds to onedimensional $\mathcal{H}_{\text {out }}$.

A quantum operation is a trace non increasing CP-map $\mathcal{T}: \mathcal{B}\left(\mathcal{H}_{\text {in }}\right) \longrightarrow \mathcal{B}\left(\mathcal{H}_{\text {out }}\right)$. In order to gather information about a quantum operation $\mathcal{T}$, the most general procedure consists in: $i)$ preparing a state $\rho \in \mathcal{B}\left(\mathcal{H}_{\text {in }} \otimes \mathcal{H}_{A}\right)$ where $\mathcal{H}_{A}$ is an ancillary system with the same dimension of $\left.\mathcal{H}_{i n} ; i i\right)$ measuring the state $\left(\mathcal{T} \otimes \mathcal{I}_{A}\right)(\rho)$ with a POVM $\left\{P_{i}\right\}$. The probability of obtaining a generic outcome $i$ is given by

$$
p_{i}=\operatorname{Tr}\left[\left(\mathcal{T} \otimes \mathcal{I}_{A}\right)(\rho) P_{i}\right]
$$

which, using the Choi-Jamiołkowski isomorphism [8],

$$
\mathcal{T}(\rho)=\operatorname{Tr}_{\text {in }}\left[\left(I_{\text {out }} \otimes \rho^{T}\right) R_{\mathcal{T}}\right], \quad R_{\mathcal{T}}=\mathcal{T} \otimes I_{\text {in }}(|I\rangle\rangle\langle\langle I|)
$$

becomes

$$
\operatorname{Tr}\left[\operatorname{Tr}_{\text {in }}\left[\left(I_{A} \otimes R_{\mathcal{T}}\right)\left(\rho^{\theta_{\text {in }}} \otimes I_{\text {out }}\right)\right] P_{i}\right]=\operatorname{Tr}\left[R_{\mathcal{T}} \Pi_{i}^{(\rho)}\right],
$$

where $\theta$ is the transposition w.r.t. the orthonormal basis in Eq. (3), and

$$
\Pi_{i}^{(\rho)}=\left\{\operatorname{Tr}_{A}\left[\left(\rho \otimes I_{\text {out }}\right)\left(I_{\text {in }} \otimes P_{i}^{\theta_{\text {out }}}\right)\right]\right\}^{T} .
$$

It is convenient to use here the notion of tester along with the theoretical framework introduced in [9]. A tester is the natural generalization of the concept of POVM from states to transformations, and is represented by a set of positive operators $\left\{\Pi_{i}\right\}$ with

$$
\sum_{i} \Pi_{i}=I \otimes \sigma, \quad \operatorname{Tr}[\sigma]=1
$$

The probability distribution in Eq. (13) is precisely represented by a Born-rule with the tester $\left\{\Pi_{i}\right\}$ in place of $\left\{P_{i}\right\}$, and the operator $R_{\mathcal{T}}$ in place of $\rho$. Such generalized Born rule can be rewritten in terms of the usual one as follows [9]

$$
p_{i}=\operatorname{Tr}\left[R_{\mathcal{T}} \Pi_{i}\right]=\operatorname{Tr}\left[\mathcal{T} \otimes \mathcal{I}(\nu) P_{i}\right]
$$

with

$$
\nu=|\sqrt{\sigma}\rangle\rangle\left\langle\langle\sqrt{\sigma}|, \quad P_{i}=\left(I \otimes \sigma^{-1 / 2}\right) \Pi_{i}\left(I \otimes \sigma^{-1 / 2}\right) .\right.
$$

This method allows a straightforward generalization of the tomographic method from states to transformation. Now tomographing a quantum operation means using a suitable tester $\Pi_{i}$ such that the expectation value of any other possible measurement can be inferred by the probability distribution $p_{i}=\operatorname{Tr}\left[R_{\mathcal{T}} \Pi_{i}\right]$. In order to achieve this task we have to require that $\left\{\Pi_{i}\right\}$ is an operator frame for $\mathcal{B}\left(\mathcal{H}_{\text {out }} \otimes \mathcal{H}_{\text {in }}\right)$. This means that we can expand any operator on $\mathcal{H}_{\text {out }} \otimes \mathcal{H}_{\text {in }}$ as follows

$$
A=\sum_{i}\left\langle\left\langle\Delta_{i} \mid A\right\rangle\right\rangle \Pi_{i} \quad A \in \mathcal{B}\left(\mathcal{H}_{\text {out }} \otimes \mathcal{H}_{\text {in }}\right) .
$$

where $\left\{\Delta_{i}\right\}$ is a possible dual of the frame $\left\{\Pi_{i}\right\}$, that is the condition $\left.\sum_{i}\left|\Pi_{i}\right\rangle\right\rangle\left\langle\left\langle\Delta_{i}\right|=I_{\text {out }} \otimes I_{\text {in }}\right.$ holds.

Optimizing the tomography of quantum operations means minimizing the statistical error in the determination of the expectation of a generic operator $A$ as in Eq. (18). This is provided by the variance

$$
\delta(A)=\sum_{i}\left|\left\langle\left\langle\Delta_{i} \mid A\right\rangle\right\rangle\right|^{2} \operatorname{Tr}\left[R_{\mathcal{T}} \Pi_{i}\right]-\left|\operatorname{Tr}\left[R_{\mathcal{T}} A\right]\right|^{2}
$$

We assume an ensemble $\mathcal{E}=\left\{R_{k}, p_{k}\right\}$ of possible transformations and a weighted set $\mathcal{G}=\left\{A_{n}, q_{n}\right\}$ of possible observables. Averaging the statistical error over these ensembles we obtain

$$
\delta_{\mathcal{E}, \mathcal{A}}:=\sum_{i}\left\langle\left\langle\Delta_{i}|G| \Delta_{i}\right\rangle\right\rangle \operatorname{Tr}\left[R_{\mathcal{E}} \Pi_{i}\right]-\sum_{k, n} p_{k} q_{n}\left|\operatorname{Tr}\left[R_{k} A_{n}\right]\right|^{2} .
$$

Optimizing this figure of merit means: $i$ ) optimizing the choice of the dual frame $\left.\left\{\Delta_{i}\right\} ; i i\right)$ optimizing the choice of the frame $\left\{\Pi_{i}\right\}$. The optimization of the set $\left\{\Pi_{i}\right\}$ reflects in both choosing the best input state for the quantum operation and the best final measurement.

In the following, for the sake of clarity we will consider $\operatorname{dim}\left(\mathcal{H}_{\text {in }}\right)=\operatorname{dim}\left(\mathcal{H}_{\text {out }}\right)=: d$, and focus on the "symmetric" case $G=I$; this happens for example when the set $\left\{A_{n}\right\}$ is an orthonormal basis, whose elements are equally weighted. Moreover, we assume that the averaged channel of the ensemble $\mathcal{E}$ is the maximally depolarizing channel, whose Choi operator is $R_{\mathcal{E}}=d^{-1} I \otimes I$.

With these assumptions the relevant term of figure of merit becomes

$$
\eta=\sum_{i}\left\langle\left\langle\Delta_{i} \mid \Delta_{i}\right\rangle\right\rangle d^{-1} \operatorname{Tr}\left[\Pi_{i}\right]
$$

Since $R_{\mathcal{E}}$ is invariant under the action of $S U(d) \times S U(d)$ we now show that it is possible to impose the same covariance also on the tester without increasing the value of $\eta$. Let us define

$$
\begin{aligned}
\Pi_{i, g, h} & :=\left(U_{g} \otimes V_{h}\right) \Pi_{i}\left(U_{g}^{\dagger} \otimes V_{h}^{\dagger}\right), \\
\Delta_{i, g, h} & :=\left(U_{g} \otimes V_{h}\right) \Delta_{i}\left(U_{g}^{\dagger} \otimes V_{h}^{\dagger}\right) .
\end{aligned}
$$

It is easy to check that $\Delta_{i, g, h}$ is a dual of $\Pi_{i, g, h}$ by evaluating the group average after the sum on $i$. Then we observe that the normalization of $\Pi_{i, g, h}$ gives

$$
\sum_{i} \int d g d h \Pi_{i, g, h}=d^{-1} I \otimes I
$$

corresponding to $\sigma=d^{-1} I$ in Eq. (17), namely one can choose $\left.\nu=d^{-1}|I\rangle\right\rangle\langle\langle I|$. In the last identity $d g$ and $d h$ are invariant measures normalized to unit.

It is easy to verify that the figure of merit for the covariant tester is the same as for the non covariant one, whence, w.l.o.g. we optimize the covariant tester. 
The condition that the covariant tester is informationally complete w.r.t. the subspace of transformations to be tomographed will be verified after the optimization.

We note that a generic covariant tester is obtained by Eq. (22), with operators $\Pi_{i}$ becoming "seeds" of the covariant POVM, and now being required to satisfy only the normalization condition

$$
\sum_{i} \operatorname{Tr}\left[\Pi_{i}\right]=d
$$

(analogous of covariant POVM normalization in [4, 10]). The problem of optimization of the dual frame has been solved in [11]. With the optimal dual, the figure of merit simplifies as

$$
\eta=\operatorname{Tr}\left[\tilde{X}^{-1}\right]
$$

where

$$
\tilde{X}=\sum_{i} \int d g d h \frac{\left.d\left|\Pi_{i, g, h}\right\rangle\right\rangle\left\langle\left\langle\Pi_{i, g, h}\right|\right.}{\operatorname{Tr}\left[\Pi_{i, g, h}\right]}=\int d g d h W_{g, h} X W_{g, h}^{\dagger}
$$

with $W_{g, h}=U_{g} \otimes U_{g}^{*} \otimes V_{h} \otimes V_{h}^{*}$ and $X=$ $\left.\sum_{i} d\left|\Pi_{i}\right\rangle\right\rangle\left\langle\left\langle\Pi_{i}\right| / \operatorname{Tr}\left[\Pi_{i}\right]\right.$. Using Schur's lemma we have [12]

$\tilde{X}=P_{1}+A P_{2}+B P_{3}+C P_{4}$,

$P_{1}=\Omega_{13} \otimes \Omega_{24}, \quad P_{2}=\left(I_{13}-\Omega_{13}\right) \otimes \Omega_{24}$,

$P_{3}=\Omega_{13} \otimes\left(I_{24}-\Omega_{24}\right), P_{4}=\left(I_{13}-\Omega_{13}\right) \otimes\left(I_{24}-\Omega_{24}\right)$,

having posed $\Omega=|I\rangle\rangle\langle\langle I| / d$ and

$$
\begin{aligned}
& A=\frac{1}{d^{2}-1}\left\{\sum_{i} \frac{\operatorname{Tr}\left[\left(\operatorname{Tr}_{2}\left[\Pi_{i}\right]\right)^{2}\right]}{\operatorname{Tr}\left[\Pi_{i}\right]}-1\right\} \\
& B=\frac{1}{d^{2}-1}\left\{\sum_{i} \frac{\operatorname{Tr}\left[\left(\operatorname{Tr}_{1}\left[\Pi_{i}\right]\right)^{2}\right]}{\operatorname{Tr}\left[\Pi_{i}\right]}-1\right\} \\
& C=\frac{1}{\left(d^{2}-1\right)^{2}}\left\{\sum_{i} \frac{d \operatorname{Tr}\left[\Pi_{i}^{2}\right]}{\operatorname{Tr}\left[\Pi_{i}\right]}-\left(d^{2}-1\right)(A+B)-1\right\} .
\end{aligned}
$$

One has

$$
\operatorname{Tr}\left[\tilde{X}^{-1}\right]=1+\left(d^{2}-1\right)\left(\frac{1}{A}+\frac{1}{B}+\frac{\left(d^{2}-1\right)}{C}\right) .
$$

We note that if the ensemble of transformations is contained in a subspace $\mathcal{V} \subseteq \mathcal{B}\left(\mathcal{H}_{\text {out }} \otimes \mathcal{H}_{\tilde{\sim}}\right)$ the figure of merit becomes $\eta=\operatorname{Tr}\left[\tilde{X}^{\ddagger} Q_{\mathcal{V}}\right]$, where $\tilde{X}^{\ddagger}$ is the MoorePenrose pseudoinverse. We now carry on the minimization for three relevant subspaces:

$\mathcal{Q}=\mathcal{B}\left(\mathcal{H}_{\text {out }} \otimes \mathcal{H}_{\text {in }}\right), \quad \mathcal{C}=\left\{R \in \mathcal{Q}, \operatorname{Tr}_{\text {out }}[R]=I_{\text {in }}\right\}$

$\mathcal{U}=\left\{R \in \mathcal{Q}, \operatorname{Tr}_{\text {out }}[R]=I_{\text {in }}, \operatorname{Tr}_{\text {in }}[R]=I_{\text {out }}\right\}$

corresponding respectively to quantum operations, general channels and unital channels. The subspaces $\mathcal{C}$ and

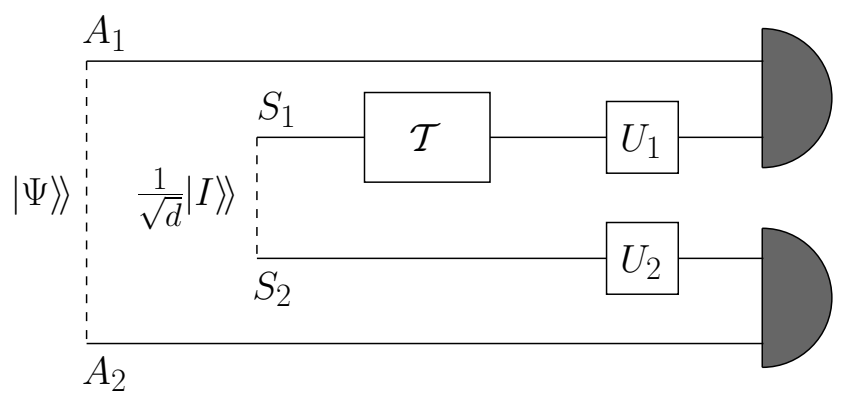

Figure 1: Physical implementation of optimal quantum transformation tomography. The two measurements are Bell's measurements preceded by a random unitary. The state $|\Psi\rangle\rangle$ depends on the prior ensemble.

$\mathcal{U}$ are invariant under the action of the group $\left\{W_{g, h}\right\}$ and thus the respective projectors decompose as

$$
Q_{\mathcal{C}}=P_{1}+P_{2}+P_{4}, \quad Q_{\mathcal{U}}=P_{1}+P_{4}
$$

Without loss of generality we can assume the operators $\left\{\Pi_{i}\right\}$ to be rank one. In fact, suppose that $\Pi_{i}$ has rank higher than 1. Then it is possible to decompose it as $\Pi=\sum_{j} \Pi_{i, j}$ with $\Pi_{i, j}$ rank 1 . The statistics of $\Pi_{i}$ can be completely achieved by $\Pi_{i, j}$ through a suitable postprocessing. For the purpose of optimization it is then not restrictive to consider rank one $\Pi_{i}$, namely $\Pi_{i}=$ $\left.\alpha_{i}\left|\Psi_{i}\right\rangle\right\rangle\left\langle\left\langle\Psi_{i}\right|\right.$, with $\sum_{i} \alpha_{i}=d$. Notice that all multiple seeds of this form lead to testers satisfying Eq. (25).

In the three cases under examination, the figure of merit is then

$$
\begin{aligned}
& \eta_{\mathcal{Q}}=\operatorname{Tr}\left[\tilde{X}^{-1}\right]=1+\left(d^{2}-1\right)\left(\frac{2}{A}+\frac{\left(d^{2}-1\right)^{2}}{1-2 A}\right) \\
& \eta_{\mathcal{C}}=\operatorname{Tr}\left[\tilde{X}^{\ddagger} Q_{\mathcal{C}}\right]=1+\left(d^{2}-1\right)\left(\frac{1}{A}+\frac{\left(d^{2}-1\right)^{2}}{1-2 A}\right) \\
& \eta_{\mathcal{U}}=\operatorname{Tr}\left[\tilde{X}^{\ddagger} Q_{\mathcal{U}}\right]=1+\left(d^{2}-1\right)\left(\frac{\left(d^{2}-1\right)^{2}}{1-2 A}\right)
\end{aligned}
$$

where $0 \leq A=\left(d^{2}-1\right)^{-1}\left(\sum_{i} \alpha_{i} \operatorname{Tr}\left[\left(\Psi_{i} \Psi_{i}^{\dagger}\right)^{2}\right]-1\right) \leq$ $\frac{1}{d+1}<\frac{1}{2}$. The minimum can simply be determined by derivation with respect to $A$, obtaining $A=1 /\left(d^{2}+1\right)$ for quantum operations, $A=1 /\left(\sqrt{2}\left(d^{2}-1\right)+2\right)$ for general channels and $A=0$ for unital channels. The corresponding minimum for the figure of merit is

$$
\begin{aligned}
& \eta_{\mathcal{Q}} \geq d^{6}+d^{4}-d^{2} \\
& \eta_{\mathcal{C}} \geq d^{6}+(2 \sqrt{2}-3) d^{4}+(5-4 \sqrt{2}) d^{2}+2(\sqrt{2}-1) \\
& \eta_{\mathcal{U}} \geq\left(d^{2}-1\right)^{3}+1
\end{aligned}
$$

The same result for quantum operations and for unital channels has been obtained in [13] in a different framework. 
These bounds are simply achieved by a single seed $\left.\Pi_{0}=d|\Psi\rangle\right\rangle\langle\langle\Psi|$, with

$$
\operatorname{Tr}\left[\left(\Psi \Psi^{\dagger}\right)^{2}\right]=\frac{2 d}{d^{2}+1}, \quad \frac{\sqrt{2}\left(d^{2}-1\right)+3}{d\left(\sqrt{2}\left(d^{2}-1\right)+2\right)}, \quad 1
$$

respectively for quantum operations, general channels and unital channels, namely with

$$
\Psi=\left[d^{-1}(1-\beta) I+\beta|\psi\rangle\langle\psi|\right]^{\frac{1}{2}}
$$

where $\beta=\sqrt{(d+1) /\left(d^{2}+1\right)}$ for quantum operations, $\beta=\left[(d-1)\left(2+\sqrt{2}\left(d^{2}-1\right)\right)\right]^{-1 / 2}$ for general channels and $\beta=0$ for unital channels, and $|\psi\rangle$ is any pure state. The informationally completeness is thus verified a posteriori (see [10]).

The same procedure can be carried on when the operator $G$ has the more general form $G=g_{1} P_{1}+g_{2} P_{2}+$ $g_{3} P_{3}+g_{4} P_{4}$, where $P_{i}$ are the projectors defined in (28). In this case Eq. (30) becomes

$$
\operatorname{Tr}\left[\tilde{X}^{-1} G\right]=g_{1}+\left(d^{2}-1\right)\left(\frac{g_{2}}{A}+\frac{g_{3}}{B}+\frac{\left(d^{2}-1\right) g_{4}}{C}\right),
$$

which can be minimized along the same lines previously followed. $G$ has this form when optimizing measuring procedures of this kind: $i$ ) preparing an input state randomly drawn from the set $\left.\left\{U_{g} \rho U_{g}^{\dagger}\right\} ; i i\right)$ measuring an observable chosen from the set $\left\{U_{h} A U_{h}^{\dagger}\right\}$.

We now show how the optimal measurement can be experimentally implemented. Referring to Fig. 1, the bipartite system carrying the Choi operator of the transformation is indicated with the labels $S_{1}$ and $S_{2}$. We prepare a pair of ancillary systems $A_{1}$ and $A_{2}$ in the joint state $|\Psi\rangle\rangle\langle\langle\Psi|$, then we apply two random unitary transformations $U_{1}$ and $U_{2}$ to $S_{1}$ and $S_{2}$, finally we perform a Bell measurement on the pair $A_{1} S_{1}$ and another Bell measurement on the pair $A_{2} S_{2}$. This experimental scheme realizes the continuous measurement by randomizing among a continuous set of discrete POVM; this is a particular application of a general result proved in [14]. The scheme proposed is feasible using e. g. the Bell measurements experimentally realized in [15]. We note that choosing $|\Psi\rangle\rangle$ maximally entangled (as proposed for example in [16]) is generally not optimal, except for the unital case.

With the same derivation starting from Eq. (21), but keeping $\operatorname{dim}\left(\mathcal{H}_{\text {in }}\right) \neq \operatorname{dim}\left(\mathcal{H}_{\text {out }}\right)$, one obtains the optimal tomography for general quantum operations. The special case of $\operatorname{dim}\left(\mathcal{H}_{i n}\right)=1$ (one has $P_{3}=P_{4}=0$ in Eq. (28) corresponds to optimal tomography of states, whereas case $\operatorname{dim}\left(\mathcal{H}_{\text {out }}\right)=1\left(P_{2}=P_{4}=0\right)$ gives the optimal tomography of POVMs. The corresponding experimental schemes are obtained by removing the upper/lower branch for POVMs/states, respectively. In the remaining branch the bipartite detector becomes a mono-partite, performing a von Neumann measurement for the qudit, preceded by a random unitary in $S U(d)$. Moreover, for the case of POVM, the state $|\Psi\rangle\rangle$ is missing, whereas, for state-tomography, both bipartite states are missing. The optimal $\eta$ in Eq. (10) is given by $\eta=d^{3}+d^{2}-d$, in both cases (for state-tomography compare with Ref. [17]).

In conclusion, we presented a general method for optimizing quantum tomography, based on the new notion of tester. The method is very versatile, allowing to consider arbitrary prior ensemble and representation. We provided the optimal experimental schemes for tomography of states and various kinds of process tomography, giving the corresponding performance, all schemes being feasible with the current technology.

[1] Quantum State Estimation, Lecture Notes in Physics, 649 M. Paris and J. Rehacek Eds., (Springer, Berlin 2004)

[2] G. M. D'Ariano, P. Lo Presti, Phys. Rev. Lett. 86, 4195 (2001).

[3] G. M. D'Ariano, L. Maccone, and P. Lo Presti Phys. Rev. Lett. 93, 250407 (2004).

[4] A. S. Holevo, Probabilistic and Statistical Aspects of Quantum Theory, North Holland, Amsterdam, 1982.

[5] E. Prugovečki, Int. J. Theor. Phys, 16321 (1977)

[6] P. Busch, Int. J. Theor. Phys., 301217 (1991).

[7] R. J. Duffin and A. C. Schaeffer, Trans. Am. Math. Soc. 72, 341 (1952); P. G. Casazza, Taiw. J. Math. 4, 129 (2000).

[8] M.-D. Choi, Lin. Alg. Phys. 10, 285 (1975).

[9] G. Chiribella, G. M. D'Ariano, P. Perinotti, arXiv: 0712.1325; M. Ziman, arXiv: 0802.3862; G. Chiribella, G. M. D'Ariano, P. Perinotti, arXiv: 0803.3237.

[10] G. M. D'Ariano, P. Perinotti, M. F. Sacchi, J. Opt. B: Quantum and Semicl. Optics, 6, S487 (2004).

[11] G. M. D'Ariano, P. Perinotti, Phys. Rev. Lett. 98, 020403 (2007).

[12] We label the four spaces in such a way that $W_{g, h} \in$ $\mathcal{B}\left(\mathcal{H}_{1} \otimes \mathcal{H}_{3} \otimes \mathcal{H}_{2} \otimes \mathcal{H}_{4}\right)$, with $\mathcal{H}_{1}=\mathcal{H}_{\text {out }}$ and $\mathcal{H}_{2}=\mathcal{H}_{\text {in }}$.

[13] A. J. Scott, J. Phys. A 41, 055308 (2008).

[14] G. Chiribella, G. M. D'Ariano, D. M. Schlingemann, Phys. Rev. Lett. 98, 190403 (2007)

[15] P. Walther, A. Zeilinger, Phys. Rev. A 72, 010302(R) (2005)

[16] M. Mohseni, A. T. Rezakhani, D. A. Lidar, Phys. Rev. A 77, 032322 (2008).

[17] A. J. Scott, J. Phys. A 39, 13507 (2006). 